\title{
Behavior of Welschinger Invariants Under Morse Simplifications
}

\author{
Erwan Brugallé(*) - Nicolas Puignau(**)
}

ABSTRACT - We relate Welschinger invariants of a rational real symplectic 4manifold before and after a Morse simplification (i.e deletion of a sphere or a handle of the real part of the surface). This relation is a consequence of a real version of Abramovich-Bertram formula which computes Gromov-Witten invariants by means of enumeration of $J$-holomorphic curves with a nongeneric almost complex structure $J$. In addition, we give some qualitative consequences of our study, for example the vanishing of Welschinger invariants in some cases.

Mathematics Subject Classification (2010). Primary 14P05, 14N10; Secondary $14 \mathrm{~N} 35,14 \mathrm{P} 25$.

KEYwORDS. Real enumerative geometry, Welschinger invariants, Gromov-Witten invariants, Symplectic sum formula

\section{Introduction}

On a rational symplectic 4-manifold $(X, \omega)$, genus 0 Gromov-Witten invariants can be computed by enumerating irreducible $J$-holomorphic rational curves on $X$, realizing a fixed homology class $d \in H_{2}(X, Z)$, and passing through a configuration of $c_{1}(X) d-1$ points, where $J$ is a generic almost complex structure on $X$ tamed by $\omega$ ([12]). Now suppose that $J$ is midly non-generic, i.e. $X$ contains a unique irreducible $J$-ho-

(*) Indirizzo dell'A.: Università Pierre et Marie Curie, Paris 6, 4 Place Jussieu, 75005 Paris, France.

E-mail: brugalle@math.jussieu.fr

(**) Indirizzo dell'A.: Universidade Federal do Rio de Janeiro, Ilha do Fundão, 21941-909 Rio de Janeiro, Brasil.

E-mail: puignau@im.ufrj.br 
lomorphic curve $E$ with $E^{2}<-1$, and moreover $E$ is a smooth rational curve with $E^{2}=-2$. In this situation, one can still compute GromovWitten invariants of $(X, \omega)$ by enumerating $J$-holomorphic curves on $(X, \omega)$, but now also taking into account reducible curves with some components mapped isomorphically to $E$. Abramovich and Bertram first proved this when $(X, \omega, J)$ is the second Hirzebruch ruled surface ([1]), Vakil extended later this proof to the case of any weak Del Pezzo surface ([14]), and eventually Ionel and Parker symplectic sum formula ([6]) provides a proof in the general case.

Results of this note are based on real versions of this AbramovichBertram type formula. A real structure $c: X \rightarrow X$ on a rational symplectic 4 -manifold $(X, \omega)$ is an involution such that $c^{*} \omega=-\omega$. The set $\mathbb{R} X=F i x(c)$ is called the real locus of $X$. Welschinger invariants provide real analogues of Gromov-Witten invariants in genus 0 for real rational symplectic 4manifolds ([15]).

Suppose that $(X, \omega, c)$ contains a real smooth rational symplectic curve $E$ with $E^{2}=-2$, and let $\left(X^{\#}, \omega^{\#}\right)$ be the symplectic sum of $(X, \omega)$ with $S^{2} \times S^{2}$ along $E$, where $E$ realizes the diagonal class in $H_{2}\left(S^{2} \times S^{2}, Z\right)$. There exist two real structures $c_{+}$and $c_{-}$on $S^{2} \times S^{2}$ for which $E$ is real, which give rise to two different real structures $c_{+}^{\#}$ and $c_{-}^{\#}$ on $\left(X^{\#}, \omega^{\#}\right)$ satisfying (with the convention that $\chi(\emptyset)=0$ )

$$
\chi\left(\mathbb{R} X_{+}^{\#}\right)=\chi(\mathbb{R} X)=\chi\left(\mathbb{R} X_{-}^{\#}\right)-2 .
$$

One may interpret this construction as follows: blow-down the real (-2)curve $E$ to a nodal real 4-manifold, and smooth the node in two different ways.

The real symplectic manifold $\left(X^{\#}, \omega^{\#}, c_{+}^{\#}\right)$ is in fact a deformation of $(X, \omega, c)$ and in this case one can immediatly extract a real version of Abramovich-Bertram formula from the complex one without decomposing $\left(X^{\#}, \omega^{\#}, c_{+}^{\#}\right)$ into a symplectic sum, as it has already been noticed by several people ([3], [4], [11], [13]). This is not true for $\left(X^{\#}, \omega^{\#}, c_{-}^{\#}\right)$, and one of the main results of this note is a real version of Abramovich-Bertram formula also in this case. These two different real versions of Abramovich-Bertram formula allows one to compare Welschinger invariants of $\left(X^{\#}, \omega^{\#}, c_{ \pm}^{\#}\right)$. This can be thought as a generalization of the invariant $\theta$ introduced by Welschinger in [15], and has several consequences (e.g. vanishing results) concerning Welschinger invariants.

Detailed proofs of the statements announced in this note will appear in [5]. 


\section{Welschinger invariants}

Let $(X, \omega, c)$ be a real rational symplectic 4-manifold, and let $J$ be an almost complex structure on $X$ tamed by $\omega$ which is $J$-antiholomorphic. Recall that the mass $m(C)$ of a real rational $J$-holomorphic curve $C$ in $(X, \omega, c)$ is the number of solitary real nodes of $\mathbb{R} C$ in $\mathbb{R} X$ (i.e. nodes locally given over $\mathbb{R}$ by the equation $x^{2}+y^{2}=0$ ). Let us fix a homology class $d$ in $H_{2}(X, Z)$, an integer $0 \leq r \leq c_{1}(X) d-1$, a connected component $S$ of $\mathbb{R} X$, and a real configuration $\underline{x}$ of $c_{1}(X) d-1$ points in $X$ containing exactly $r$ points in $S$ and $\frac{c_{1}(X) d-1-r}{2}$ pairs of complex conjugated points. When $J$ is generic, Welschinger proved in [15] that the number of irreducible real rational $J$-holomorphic curves $C$, counted with multiplicity $(-1)^{m(C)}$, incident to $\underline{x}$ and realizing the class $d$ is finite and depends only on $d$ and $r$. This number is a Welschinger invariant of $(X, \omega, c)$, and we denote it by $W_{\mathbb{R} X, S}(d, r)$. We omit the reference to $S$ when $S=\mathbb{R} X$, or to $r$ when $r=c_{1}(X) d-1$.

Suppose now that $J$ is mildly non-generic as above, in particular the ( -2 )-curve $E$ is real. Counting real rational $J$-holomorphic curves in $X$ with multiplicity $(-1)^{m(C)}$ does not give a number depending only on $d$ and $r$, since $J$ is non-generic ([15], [7]).

DEFINITION 2.1. Let C be a nodal real rational J-holomorphic curve in $X$ intersecting the (-2)-curve $E$ transversally. We denote respectively by $\alpha$ and $\beta$ the number of real and pairs of complex conjugated intersection points in $C \cap E$. For any integer $k \geq 0$, we define the two kth multiplicities of $C$ as follows:

$$
\mu_{k}^{+}(C)=(-1)^{m(C)} \sum_{k=\alpha_{k}+2 \beta_{k}}\left(\begin{array}{c}
\alpha \\
\alpha_{k}
\end{array}\right)\left(\begin{array}{c}
\beta \\
\beta_{k}
\end{array}\right)
$$

and

$$
\mu_{k}^{-}(C)= \begin{cases}(-1)^{m(C)+\beta} 2^{\beta} & \text { if } \alpha=0 \text { and } k=\beta ; \\ 0 & \text { otherwise. }\end{cases}
$$

As above choose $d \in H_{2}(X, Z)$, an integer $0 \leq r \leq c_{1}(X) d-1$, a connected component $S$ of $\mathbb{R} X \backslash \mathbb{R} E$, and a generic real configuration $\underline{x}$ of $c_{1}(X) d-1$ points in $X$ containing exactly $r$ points in $S$ and $\frac{c_{1}(X) d-1-r}{2}$ pairs of complex conjugated points. For each integer $k \geq 0$, we denote by $\mathcal{R}_{k}(d, \underline{x})$ the set of all irreducible rational real $J$-holomorphic curves in $X$ passing through all points in $\underline{x}$ and realizing the class $d-k E$. The set 
$\mathcal{R}_{k}(d, \underline{x})$ is finite, and any curve in $\mathcal{R}_{k}(d, \underline{x})$ is nodal and intersects $E$ transversally. Moreover $\mathcal{R}_{k}(d, \underline{x})$ is non-empty only for finitely many values of $k$. We define the two following numbers:

$$
W_{\mathbb{R} X, S}^{ \pm}(d, r)=\sum_{k \geq 0} \sum_{C \in \mathcal{R}_{k}(d, \underline{x})} \mu_{k}^{ \pm}(C) .
$$

Let $\left(X^{\#}, \omega^{\#}, c^{\#}\right)$ be as above with $c^{\#}=c_{ \pm}^{\#}$, and let $S^{\#}$ be the component of $\mathbb{R} X^{\#}$ containing the deformation of $S$. Note that the homology groups $H_{2}(X, Z)$ and $H_{2}\left(X^{\#}, Z\right)$ are canonically identified ([6]).

THEOREM 2.2. Under the above hypotheses, one has:

(i) if $\chi\left(\mathbb{R} X^{\#}\right)=\chi(\mathbb{R} X)$, then

$$
W_{\mathbb{R} X^{\#}, S^{\#}}(d, r)=W_{\mathbb{R} X, S}^{+}(d, r) ;
$$

(ii) if $\chi\left(\mathbb{R} X^{\#}\right)=\chi(\mathbb{R} X)+2$, then

$$
W_{\mathbb{R} X^{\#}, S^{\#}}(d, r)=W_{\mathbb{R} X, S}^{-}(d, r) .
$$

As an immediate consequence of Theorem 2.2, the numbers $W_{\mathbb{R} X, S}^{ \pm}(d, r)$ depend only on $d$ and $r$. As mentioned in the introduction, part $(i)$ in Theorem 2.2 is an immediate consequence of Abramovich-Bertram formula and was known before ([3], [4], [11], [13]).

\section{Applications}

Here we announce some consequences of Theorem 2.2, in particular when $X$ is $\mathrm{C} P_{6}^{2}$, the complex projective plane $\mathrm{C} P^{2}$ blown up in 6 points.

\section{1 - Computation for degree 6 curves with 6 fixed nodes}

Let us also denote by $\widetilde{\mathrm{C} P_{6}^{2}}$ the projective plane $\mathrm{C} P^{2}$ blown up at 6 points lying on a smooth conic $E$. Here we enumerate real rational curves realizing twice the anti-canonical class $\delta=2 c_{1}\left(\mathrm{CP}_{6}^{2}\right)^{\vee}$ in $\mathrm{CP}_{6}^{2}$ and $\mathrm{C} P_{6}^{2}$.

Given a real structure on $\widetilde{C P_{6}^{2}}$, we denote by $\widetilde{R P_{6}^{2}}$ its real part. Note that $\widetilde{R P} P_{6}^{2}$ is not necessarily $\mathbb{R} P^{2}$ blown up in 6 real points lying on a conic. Given a generic configuration $\underline{x}$ of $c_{1}\left(\mathrm{CP}_{6}^{2}\right) \delta-1=5$ real points in $\widehat{\mathrm{C} P}{ }_{6}^{2}$, we set $n_{\chi\left(\mathbb{R} P_{6}^{2}\right)}^{\sim}(\delta-k E):=\sum_{C \in \mathcal{R}_{k}(\delta, \underline{x})} \mu_{k}^{ \pm}(C)$. 
Proposition 3.1. For any choice of $S$, there exists a configuration of 5 real points in $\widehat{\mathrm{CP}}{ }_{6}^{2}$ such that:

\begin{tabular}{c|c|c|c|c|c|c|c|c} 
& $n_{-5}^{+}$ & $n_{-5}^{-}$ & $n_{-3}^{+}$ & $n_{-3}^{-}$ & $n_{-1}^{+}$ & $n_{-1}^{-}$ & $n_{1}^{+}$ & $n_{1}^{-}$ \\
\hline$\delta$ & 522 & 522 & 236 & 236 & 78 & 78 & 0 & 0 \\
\hline$\delta-E$ & 472 & 0 & 280 & 0 & 152 & 0 & 72 & 0 \\
\hline$\delta-2 E$ & 6 & 0 & 6 & 0 & 6 & 0 & 6 & 0 \\
\hline
\end{tabular}

COROLlary 3.2. The surface $\mathrm{CP}_{6}^{2}$ has the following Welschinger invariants:

\begin{tabular}{c|c|c|c|c|c}
$\chi\left(\mathbb{R} P_{6}^{2}\right)$ & -5 & -3 & -1 & 1 & 3 \\
\hline$W_{\mathbb{R} P_{6}^{2}, S}(\delta)$ & 1000 & 522 & 236 & 78 & 0
\end{tabular}

The value $W_{\mathbb{R} P_{6}^{2}}(\delta)$ when $\chi\left(\mathbb{R} P_{6}^{2}\right)=-5$ has been first computed by the first author ([3], [4]). The numbers $W_{\mathbb{R} P_{6}^{2}}(\delta)$ when $\chi\left(\mathbb{R} P_{6}^{2}\right)=-3,-1,1$, as well as $W_{\mathbb{R} P^{2} \sqcup S^{2}, \mathbb{R} P^{2}}(\delta)$ have been first computed by Itenberg, Kharlamov and Shustin ([10]). The vanishing of $W_{\mathbb{R} P^{2} \sqcup S^{2}, S}(\delta)$ is actually a general fact:

Proposition 3.3. If $(X, \omega, c)$ is a real symplectic 4-manifold with disconnect real part, then for any $d \in H_{2}(X, Z)$, any $r \geq 2$, and any choice of $S$, one has

$$
W_{\mathbb{R} X, S}(d, r)=0 .
$$

3.2 - Behavior of purely real Welschinger invariants with respect to Euler characteristic

Given a real toric Del Pezzo surface $X$ equipped with its tautological real toric structure and a class $d \in H_{2}(X, Z)$, one has ([8])

$$
W_{\mathbb{R} X}(d) \geq W_{\mathbb{R} X}\left(d, c_{1}(X) d-3\right) .
$$

Theorem 2.2 provides a natural generalization of this formula in the particular cases when $X$ is $S^{2} \times S^{2}$ or $\mathrm{C} P_{6}^{2}$.

THeOREm 3.4. Let $\left(X_{1}, \omega_{1}\right)$ and $\left(X_{2}, \omega_{2}\right)$ be two symplectic 4-manifolds deformation equivalent to either $\mathrm{C} P^{1} \times \mathrm{CP}^{1}$ or $\mathrm{C} P_{6}^{2}$ equipped with their standard symplectic form. Choose a real structure $c_{1}$ on $X_{1}$, and a real structure $c_{2}$ on $X_{2}$. Then for any $d \in H_{2}(X, Z)$, one has

$$
W_{\mathbb{R} X_{1}, S_{1}}(d) \geq W_{\mathbb{R} X_{2}, S_{2}}(d) \quad \text { if } \quad \chi\left(\mathbb{R} X_{1}\right) \leq \chi\left(\mathbb{R} X_{2}\right) .
$$


Note that Theorem 3.4 does not generalize immediately to any symplectic 4-manifold. Indeed, according to [2] one has $W_{\mathbb{R} P^{2}}(9,2)<W_{\mathbb{R} P^{2}}(9,0)$, i.e. Theorem 3.4 does not hold in the case of $\mathrm{CP}^{2}$ blown up in 26 points.

\section{3 - Modified Welschinger invariants}

In the case when $\mathbb{R} X$ is not connected, one may slightly modify the definition of Welschinger invariants given in section 2. Namely, given $S$ a connected component of $\mathbb{R} X$, the modified mass of a real rational curve $C$ is defined as the number of solitary real nodes of $C$ lying in $S$. Counting real curves with this sign produces a new invariant, denoted by $\widetilde{W}_{\mathbb{R} X, S}$.

Our method also allows us to compute these invariants in the case of $\mathrm{C} P_{6}^{2}$. In particular we have the following two propositions.

Proposition 3.5. $\quad \widetilde{W}_{\mathbb{R} P^{2} \sqcup S^{2}, \mathbb{R} P^{2}}(\delta)=160$ and $\widetilde{W}_{\mathbb{R} P^{2} \sqcup S^{2}, S^{2}}(\delta)=96$.

The value of $\widetilde{W}_{\mathbb{R} P^{2} \sqcup S^{2}, \mathbb{R} P^{2}}(\delta)$ has been first computed by Itenberg, Kharlamov and Shustin ([10]).

Proposition 3.6. For any class $d \in H_{2}\left(\mathrm{CP}_{6}^{2}, \mathrm{Z}\right)$, we have

$$
\widetilde{W}_{\mathbb{R} P^{2} \sqcup S^{2}, \mathbb{R} P^{2}}(d) \geq \widetilde{W}_{\mathbb{R} P^{2} \sqcup S^{2}, S^{2}}(d) \geq 0 .
$$

The positivity of $\widetilde{W}_{\mathbb{R} P^{2} \sqcup S^{2}, R P^{2}}(d)$ whenever $d$ contains a real algebraic curve has first been established in [10].

\section{4 - Relation to tropical Welschinger invariants of $\mathrm{F}_{2}$}

We end this note relating some tropical Welschinger invariants of $\mathrm{F}_{2}$ to genuine Welschinger invariants of the quadric ellipsoid $Q$. The only real homology classes of $Q$ are multiple of the hyperplane section $h$. We say that a tropical curve in $\mathbb{R}^{2}$ is of class $a B+b F$ in ${ }^{T F}$ if its Newton polygon has vertices $(0,0),(0, a),(b, a)$, and $(2 a+b, 0)$. We denote by $W_{\mathrm{TF}_{2}}(d B)$ the irreducible tropical Welschinger invariant of $\mathrm{TF}_{2}$ for curves of class $d B([9])$.

Proposition 3.7. For any positive integer d

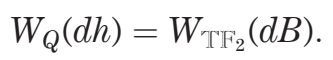


Acknowledgments. This final formulation of our results wouldn't have been possible without the patient explanations of many of our colleagues. We are in particular indebted to Simone Diverio, Ilia Itenberg, Viatcheslav Kharlamov, Frédéric Mangolte, Christian Peskine, Brett Parker, Patrick Popescu, and Jean-Yves Welschinger.

Both authors were supported by the Brazilian-French Network in Mathematics. E.B. is also partially supported by the ANR-09-BLAN-003901 and ANR-09-JCJC-0097-01.

\section{REFERENCES}

[1] D. Abramovich - A. Bertram, The formula $12=10+2 \times 1$ and its generalizations: Counting rational curves on $\mathrm{F}_{2}$. Contemporary Math.,276 (2001), pp.83-88.

[2] A. Arroyo - E. Brugallé - L. Lopez de Medrano, Recursive formulas for Welschinger invariants, Int. Math. Res. Not., No. 5 (2011), pp. 1107-1134.

[3] E. BRUGALLÉ, Floor diagrams relative to a conic. "Real structures on complex varieties : new results and perspectives" conference, CIRM, June 2010.

[4] E. BRUGALLÉ, Enumeration of tropical curves in tropical surfaces. Oberwolfach report "Real Enumerative Questions in Complex and Tropical Geometry" workshop, March 2011.

[5] E. Brugallé - N. Puignau, Behavior of Welschinger invariants under Morse simplifications. In preparation.

[6] E.-N. IONEL - T. H. PARKer, The symplectic sum formula for Gromov-Witten invariants. Ann. of Math. 159 (2), no. 3 (2004), pp. 935-1025.

[7] I. ITENBERG - V. KHARLAMOV - E. SHUSTIN, Welschinger invariant and enumeration of real rational curves. Int. Math. Research Notices, 49 (2003), pp. 2639-2653.

[8] I. Itenberg - V. Kharlamov - E. Shustin, Logarithmic equivalence of Welschinger and Gromov-Witten invariants. Russian Math. Surveys, 59 (6) (2004), pp. 1093-1116.

[9] I. Itenberg - V. Kharlamov - E. Shustin, A Caporaso-Harris type formula for Welschinger invariants of real toric Del Pezzo surfaces. Comment. Math. Helv., 84 (2009), pp. 87-126.

[10] I. ItENBERG - V. KHARLAMov - E. Shustin, Welschinger invariants of real Del Pezzo surfaces of degree $\geq 3$. arXiv:1108.3369.

[11] V. KHARLAMOV, Remarks on recursive enumeration of real rational curves on Del Pezzo surfaces. "Genève-Paris-Strasbourg Tropical geometry" seminar, IRMA, November 2010.

[12] D. McDuff - D. Salamon, J-holomorphic curves and symplectic topology. Am. Math. Soc. Coll. Publi. volume 52 (2004).

[13] R. Rasdeaconu - J. Solomon, Relative open Gromov-Witten invariants. In preparation.

[14] R. VAKIL, Counting curves on rational surfaces. Manuscripta Math. 102 (2000), pp. 53-84.

[15] J.-Y. WeLSChInger, Invariants of real symplectic 4-manifolds and lower bounds in real enumerative geometry. Invent. Math., 162 (1) (2005), pp. 195-234.

Manoscritto pervenuto in redazione 22 Marzo 2012. 
